\title{
Diversity and evolution of the endosymbionts of Bemisia tabaci in China
}

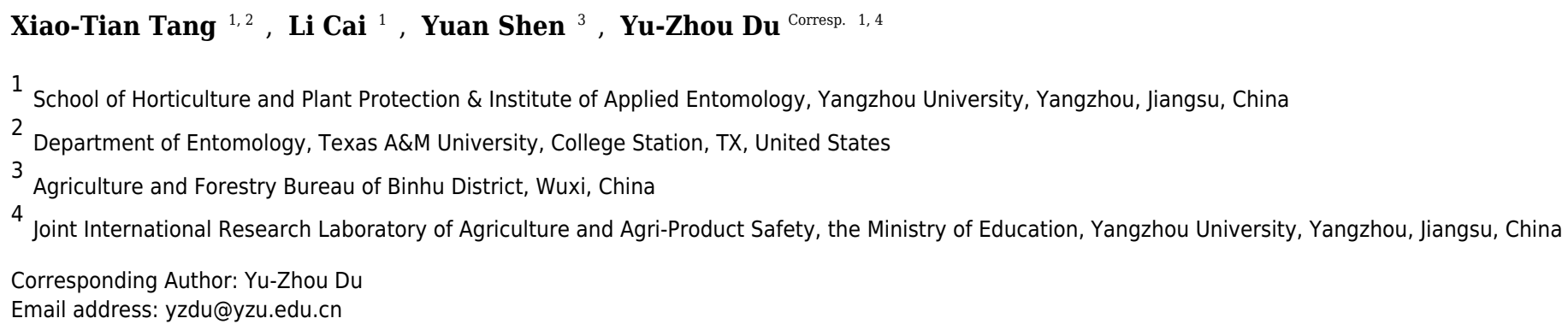

The whitefly Bemisia tabaci (Gennadius) (Hemiptera: Aleyrodidae) is a cryptic species complex, including members that are pests of global importance. This study presents a screening of $B$. tabaci species in China for infection by the primary endosymbiont, Portiera aleyrodidarum, and two secondary endosymbionts, Arsenophonus and Cardinium . The results showed that $P$. aleyrodidarum was detected in all $B$. tabaci individuals, while Arsenophonus was abundant in indigenous species of $B$. tabaci Asia II 1, Asia II 3 and China 1 but absent in the invasive species, Middle East-Asia Minor 1 (MEAM1); Cardinium presented in the Mediterranean (MED), Asia II 1 and Asia II 3 species but was rarely detected in the MEAM1 and China 1 species. Moreover, phylogenetic analyses revealed that the $P$. aleyrodidarum and mtCO1 (mitochondrial cytochrome oxidase 1) phylograms were similar and corresponding with the five distinct cryptic species clades to some extent, probably indicating an ancient infection followed by vertical transmission and subsequent co-evolutionary diversification. In contrast, the phylogenetic trees of Arsenophonus and Cardinium were incongruent with the mtCO1 phylogram, potentially indicating horizontal transmission in $B$. tabaci cryptic species complex. Taken together, our study showed the distinct infection status of endosymbionts in invasive and indigenous whiteflies; we also probably indicated the co-evolution of primary endosymbiont and its host as well as potential horizontal transfer of secondary endosymbionts. 
1 Diversity and evolution of the endosymbionts of Bemisia

2 tabaci in China

3

4 Xiao-Tian Tang ${ }^{1,3,}$, Li Cai ${ }^{1, *}$, Yuan Shen ${ }^{1,4}$, Yu-Zhou Du ${ }^{1,2 * *}$

5

$6{ }^{1}$ School of Horticulture and Plant Protection \& Institute of Applied Entomology, Yangzhou

7 University, Yangzhou, Jiangsu, 225009, China

8 2Joint International Research Laboratory of Agriculture and Agri-Product Safety, the Ministry of

9 Education, Yangzhou University, Yangzhou 25009, China.

$10{ }^{3}$ Current address: Department of Entomology, Texas A\&M University, College Station, TX

1177843 , USA

$12{ }^{4}$ Agriculture and Forestry Bureau of Binhu District, Wuxi 214071, China

*These authors contributed equally to this work

**Corresponding author: School of Horticulture and Plant Protection \& Institute of Applied

16 Entomology, Yangzhou University, 48 Wenhui Road (East), Yangzhou, Jiangsu, 225009, China.

17 Tel: 0086-514-87971854; Fax: 0086-514-87347537.

18 E-mail address: yzdu@yzu.edu.cn (YZD) 
19

20

21

22

23

24

25

26

27

28

29

30

31

\section{Abstract}

The whitefly Bemisia tabaci (Gennadius) (Hemiptera: Aleyrodidae) is a cryptic species complex, including members that are pests of global importance. This study presents a screening of $B$. tabaci species in China for infection by the primary endosymbiont, Portiera aleyrodidarum, and two secondary endosymbionts, Arsenophonus and Cardinium. The results showed that $P$. aleyrodidarum was detected in all B. tabaci individuals, while Arsenophonus was abundant in indigenous species of B. tabaci Asia II 1, Asia II 3 and China 1 but absent in the invasive species, Middle East-Asia Minor 1 (MEAM1); Cardinium presented in the Mediterranean (MED), Asia II 1 and Asia II 3 species but was rarely detected in the MEAM1 and China 1 species. Moreover, phylogenetic analyses revealed that the P. aleyrodidarum and $m t C O 1$ (mitochondrial cytochrome oxidase 1) phylograms were similar and corresponding with the five distinct cryptic species clades to some extent, probably indicating an ancient infection followed by vertical transmission and subsequent co-evolutionary diversification. In contrast, the phylogenetic trees of Arsenophonus and Cardinium were incongruent with the $m t C O 1$ phylogram, potentially indicating horizontal transmission in B. tabaci cryptic species complex. Taken together, our study showed the distinct infection status of endosymbionts in invasive and indigenous whiteflies; we also probably indicated the co-evolution of primary endosymbiont and its host as well as potential horizontal transfer of secondary endosymbionts. 


\section{Introduction}

Bemisia tabaci is a cryptic species complex comprising a minimum of 40 morphologically similar species (De Barro et al. 2011; Dinsdale et al. 2010; Hu et al. 2018; Wang et al. 2017). Among members of the complex, the Middle East-Asia Minor 1 (MEAM1) and Mediterranean (MED) groups (commonly known as the B and Q biotypes, respectively) have drawn much attention due to their global invasion and vectoring important plant pathogens (e.g. Tomato yellow leaf curl virus, TYLCV) (Brown 1994; Cohen \& Nitzany 1966). In China, B. tabaci was not considered as a serious pest until the arrival of the MEAM1 group in the mid-1990s (Qiu et al. 2007). In 2004, the MED group was detected, and rapidly became widely distributed in China, causing considerable damage to a wide range of vegetables, fibers, and ornamental crops (Chu et al. 2005). It is interesting that MED has been replacing the earlier invader MEAM1 as well as several indigenous species of whiteflies (e.g. Asia II and China 1) in many regions (Liu et al. 2007; Sun et al. 2013).

Associations between insects and endosymbionts are quite common in nature. It has been estimated that at least $15-20 \%$ of all insect species live in symbiotic relationships with bacteria (Douglas 1998; Gosalbes et al. 2010). Endosymbionts associated with insects can be classified into primary endosymbionts (P-endosymbionts) and secondary endosymbionts (S-endosymbionts) (Baumann 2005). The P-endosymbionts are obligate and usually have mutualist relationships with their hosts. Besides, P-endosymbionts are generally localized in bacteriocytes of bacteriome (Caspi-Fluger et al. 2011) and transmitted vertically from mother to progeny (Werren \& O’Neill 1997). In contrast, the S-endosymbionts are usually facultative symbionts, and they could reside in several host tissues such as gut, hemolymph, Malpighian tubules, salivary glands or ovarian cells (Cicero et al. 2016; Cooper et al. 2014; Dobson et al. 1999; Zchori-Fein et al. 1998). Infection of secondary endosymbionts can be either maternally inherited or horizontally-transmitted (Moran 
\& Baumann 2000). It has been discovered that the P-endosymbionts Portiera aleyrodidarum and S-endosymbionts such as Arsenophonus, Cardinium, Fritschea, Hamiltonella, Rickettsia, and Wolbachia were infected in whiteflies (Bing et al. 2013; Chiel et al. 2007; Chu et al. 2011; Everett et al. 2005; Karut et al. 2017; Thao \& Baumann 2004; Zchori-Fein et al. 2014). Previous studies have investigated the prevalence, diversity and evolution of endosymbionts in the B. tabaci species complex from different countries or regions (e.g. Turkey, China, Brazil and Africa) (Ahmed et al. 2010; Bing et al. 2013; Bing et al. 2014; Ghosh et al. 2015; Hashmi et al. 2018; Jahan et al. 2015; Karut et al. 2017; Marubayashi et al. 2014; Santos-Garcia et al. 2015; Sseruwagi et al. 2018; Thierry et al. 2011; Thierry et al. 2015). However, most of these reports only focused on the two invasive cryptic species MEAM1 and MED; furthermore, for studies from China, the sample size and distribution range were limited. For example, regarding prevalence of S-endosymbionts in $B$. tabaci, only the laboratory samples from two provinces were investigated (Bing et al. 2013); only one S-endosymbiont (Wolbachia) was explored from a slightly larger geographical scale (Bing et al. 2014).

Therefore, although substantial datasets regarding endosymbionts prevalence of $B$. tabaci species complex are present, their current situation in whole China is still not very clear and further investigation is essential. In the present study, our first goal is to investigate the prevalence and diversity of the P-endosymbionts $P$. aleyrodidarum and two common S-endosymbionts Arsenophonus and Cardinium (as representatives of S-endosymbionts) within B. tabaci in wider range of China. We also aim to explore the evolutionary relationships between these three endosymbionts and their host based on phylogenetic analyses of $16 \mathrm{~S}$ and 23S ribosomal DNA $(r D N A)$ (from endosymbionts) as well as mitochondrial cytochrome oxidase 1 ( $m t C O 1)$ gene (from B. tabaci). Our study will not only uncover the current status of endosymbionts infection within B. tabaci in China, but also greatly provide a supplement for studies of $B$. tabaci endosymbionts worldwide.

\section{Materials and Methods}


99

100

101

102

103

104

105

106

107

108

109

110

111

112

113

114

115

116

117

118

119

120

121

122

123

124

125

\section{Sample collection}

Totally 1,510 B. tabaci individuals were collected from 71 geographical locations, including 19 provinces and four municipalities in China (Fig. 1). At each location, B. tabaci was collected from different leaves of separate plants. The collection details, geographical sites and host plants were described in Table S1.

\section{DNA extraction and gene amplification}

Total DNA was extracted from individual whitefly as described in Luo et al. (2002). The primers of $m t C O 1$ gene was used for whitefly species identification. $16 \mathrm{~S} r D N A$ primers were used to detect $P$. aleyrodidarum and Cardinium, and for Arsenophonus, 23S rDNA primers were utilized. The primers, annealing temperature, and predicted PCR products size were shown in Table 1. The PCR reaction mixture contained 1 U Taq DNA polymerase, $5 \mu 1(10 \times)$ reaction buffer, $3 \mu \mathrm{MgCl}_{2}$ (final concentration of $\left.25 \mathrm{mmol} / \mathrm{L}\right), 2 \mu \mathrm{dNTPs}(10 \mathrm{mmol} / \mathrm{L}), 2 \mu 1$ of forward and reverse primers $(20 \mu \mathrm{mol} / \mathrm{L}$ each) and $2 \mu \mathrm{l}$ of template DNA (Simon et al. 1994). PCR products were visualized by $1.5 \%$ agarose gels and sequenced by IGE Biotechnology Co., Ltd (Guangzhou, China). The sequences were deposited in Genbank under accession numbers KP137471 to KP137491 for B. tabaci mtCO1 gene, KP201110 to KP201126 for P. aleyrodidarum 16S rDNA, KP201103 to KP201109 for Arsenophonus 23S rDNA, and KP201127 to KP201134 for Cardinium 16S rDNA (Table 2).

\section{Sequence alignment and phylogenetic analysis}

Sequence fragments were assembled using ContigExpress and aligned using the Clustal X 1.83 program (Chenna et al. 2003). The GenBank database was searched for homologous sequences of $m t C O 1,16 \mathrm{~S}$ rDNA and 23S rDNA using the Basic Local Alignment Search Tool (BLAST). Phylogenetic trees were constructed using MrBayes 3.2.1 (Ronquist \& Huelsenbeck 2003). The best-fit substitution model for each of the aligned sequences was selected with the program Modeltest 3.7 (Posada \& Crandall 1998). All the trees were constructed using the GTR $+\mathrm{I}+\mathrm{G}$ 
126

127

128

129

130

131

132

133

134

135

136

137

138

139

140

141

142

143

144

145

146

147

148

149

150

151

152

model. The Metropolis-coupled Markov chain Monte Carlo algorithm was conducted using four chains. Analyses were initiated with random starting trees, processed for $3 \times 10^{6}$ generations, and sampled every 1,000 generations. For the burn-in period, we discarded 100,000 generations. Posterior clade probabilities obtained from the analysis were used to assess nodal support. Tree information was visualized and edited using FigTree ver. 1.3.1 (http://tree.bio.ed.ac.uk/software/figtree/).

\section{Results}

\section{Molecular identification of $\boldsymbol{B}$. tabaci individuals}

Analyses of $m t C O 1$ sequences indicated that our 1,510 B. tabaci samples comprised of two invasive species (MEAM1 and MED) and three indigenous species (Asia II 1, Asia II 3 and China 1). Among the individuals tested, $36.4 \%(550 / 1,510)$ and $39.7 \%(600 / 1,510)$ were identified as MEAM1 and MED, respectively. The remained 4.6\%, 9.3\% and 9.9\% insects were identified as Asia II 1 (70/1,510), Asia II 3 (140/1,510) and China 1 (150/1,510), respectively (Fig. 1A; Table S1). Moreover, both the MEAM1 and MED whiteflies were widely-distributed across China, whereas the three indigenous species (Asia II 1, Asia II 3 and China 1) were relatively less detected and mainly distributed in southeastern part of China (Fig. 1B).

\section{Prevalence of endosymbionts among five species of $B$. tabaci}

As expected, P. aleyrodidarum was detected in all whitefly individuals and species (Fig. 2A); 21.7\% (327/1,510) individuals harbored S-endosymbiont (Arsenophonus or Cardinium); 5.6\% $(84 / 1,510)$ whiteflies were co-infected with both, and the remained majority $(72.8 \% ; 1,099 / 1,510)$ lacked an infection with either of the two S-endosymbionts. Infection frequencies with Sendosymbionts varied across B. tabaci species. In detail, Arsenophonus was abundant in the indigenous species (Asia II 1, Asia II 3 and China 1), with infection rates ranging from 50.7-55.7\%; however, it was infrequent in MED (11.5\%) and MEAM1 (0.0\%) (Fig. 2B). Cardinium was moderately common in MED, Asia II 1 and Asia II 3 populations with frequencies of 20.3-40.7\%, 
153 but rarely detected in MEAM1 (0.5\%) and not found in China 1 (Fig. 2C).

154

155

156

157

158

159

160

161

162

163

164

165

166

167

168

169

170

171

172

173

174

175

176

177

178

179

\section{Genetic diversity of $B$. tabaci and its endosymbionts}

Aligned sequences from $B$. tabaci (813 bp, mtCO1 gene), P. aleyrodidarum (886 bp, 16s rDNA), Arsenophonus (551 bp, 23S rDNA), and Cardinium (460 bp, 16S rDNA) were used to analyze the genetic variation of whitefly and its endosymbionts. The results showed that 21 haplotypes were identified in whiteflies based on $m t C O 1$ sequences, while 17, 7 and 8 symbiont haplotypes were defined based on analysis of P. aleyrodidarum, Arsenophonus and Cardinium sequences, respectively (Table 2). These haplotypes sequences were used to construct the phylogenetic trees.

\section{Phylogenetic analysis of $B$. tabaci and endosymbionts}

Phylogenetic trees were constructed for B. tabaci, P. aleyrodidarum, Arsenophonus and Cardinium based on $m t C O 1$ gene, 16s $r D N A, 23 \mathrm{~S} r D N A$ and $16 \mathrm{~s} r D N A$ sequences of haplotypes, respectively. We also pulled the related sequences from NCBI to explore the phylogenetic status of our haplotypes. The GenBank number of those related sequences could be found in phylogenetic trees. Based on the $m t C O 1$ gene, we found five distinct genetic groups of $B$. tabaci, which was corresponding with the five cryptic species including the MEAM1, MED, Asia II 1, Asia II 3 and China 1 (Fig. 3). We can also find that all of our MED individuals belonged to Q1 subclade (Chu et al. 2011). For phylogenetic trees of endosymbionts, several distinct bacterial strains existed within individual bacterium. It is interesting that the phylogenetic tree of $P$. aleyrodidarum were similar to the $m t C O 1$ tree and exhibited four groups corresponding to MEAM1+MED, Asia II 1, Asia II 3 and China 1 clades to some extent (Fig. 4). While there are still several differences. For example, the sequences of $P$. aleyrodidarum of China 1 are not well clustered and $P$. aleyrodidarum from B. tabaci species MED (KP201113) was also present in that China 1 clade. In addition, the MEAM1+MED clade (Fig. 4) contained sequences of $P$. aleyrodidarum from both MEAM1 and MED; however, it was separated into two distinct clades in the B. tabaci tree (Fig. 
180

181

182

183

184

185

186

187

188

189

190

191

192

193

194

195

196

197

198

199

200

201

202

203

204

205

206

3). In contrast, the phylogenetic trees for Arsenophonus and Cardinium were totally incongruent with the $m t C O 1$ tree, exhibiting four (A1-A4) and three groups (C1-C3), respectively (Fig 5 and $6)$.

\section{Discussion}

In this study, we conducted an extensive screening of $B$. tabaci for the presence one Pendosymbiont of two common S-endosymbionts, along with phylogenetic analyses of these symbionts to compare with host species from the cryptic B. tabaci complex. The reason we chose Arsenophonus and Cardinium as representatives of S-endosymbionts because they are the very common S-endosymbionts in whiteflies, and Wolbachia has been thoroughly investigated in the study of Bing et al. (2014). The results showed that P-endosymbiont $P$. aleyrodidarum was detected in all whitefly individuals while S-endosymbionts infection were varied in species. The variation in the prevalence of endosymbionts could be influenced by numerous factors such as host, environmental conditions, geographical location or even climate (Chu et al. 2011; Karut \& Tok 2014; Morag et al. 2012; Skaljac et al. 2010). In our study, Arsenophonus was abundant in Asia II 1, Asia II 3 and China 1 species but absent in the invasive species MEAM1, which is exactly consistent with previous studies (Bing et al. 2013; Karut et al. 2017); Cardinium was present in the MED, Asia II 1and Asia II 3 species (20.3-40.7\%) but was rarely detected in MEAM1 and not detected in China 1. Taken together, it seemed that these two S-endosymbionts had high prevalence in native species rather than invasive species, which is consistent with another S-endosymbiont Wolbachia but in contrast to Hamiltonella; Hamiltonella was found abundant in invasive species rather than native species (Bing et al. 2013).

Previous studies showed that B. tabaci could be co-infected with particular pairs of Sendosymbionts, including Rickettsia and Hamiltonella, Hamiltonella and Cardinium, or Rickettsia and Arsenophonous; others were less common, such as Cardinium and Rickettsia, Hamiltonella and Arsenophonous, Cardinium and Wolbachia, and even three or four endosymbionts together (Gueguen et al. 2010; Karut \& Tok 2014; Marubayashi et al. 2014; Pan et al. 2012; Skaljac et al. 
207

208

209

210

211

212

213

214

215

216

217

218

219

220

221

222

223

224

225

226

227

228

229

230

231

232

233

2010). In our study, we found evidence for a low rate (5.6\%) of co-infection with Arsenophonous and Cardinium in four B. tabaci species (MEAM1 was the exception since Arsenophonous was not detected in this species), which has also been reported before (Bing et al. 2013; Chu et al. 2011; Parrella et al. 2014; Zchori-Fein et al. 2014). However, the reason of so few rate of co-infection by Arsenophonous and Cardinium is that Arsenophonous and Cardinium are potential reproductive manipulators that compete for resources inside the bacteriocytes, thus compromising the fitness of host (Gottlieb et al. 2008). We have one plausible explanation for the co-infection status, that is Cardinium is not restricted to the bacteriocytes (Skaljac et al. 2010), and perhaps the non-overlapping niche makes co-infection of Arsenophonous and Cardinium possible. In addition, the co-infection symbiont system in whiteflies may indicate the roles of dual endosymbionts: work as important mutually dependents to provide full complement of nutrients to their host (Rao et al. 2015) or affect the fitness and biology of the B. tabaci (Ghosh et al. 2018).

Our phylogenetic analyses indicated that B. tabacia mtCO1 sequences could be assigned to five distinct clades, which conformed to existing MEAM1, MED, Asia II 1, Asia II 3, and China 1 clades. Similarly, the sequences of the P-endosymbionts $P$. aleyrodidarum were assigned to their own clade and the phylogeny was similar with that of $B$. tabaci genetic groups to some extent. This may potentially indicate an ancient infection followed by vertical transmission and subsequent co-evolutionary diversification (Baumann 2005). Meanwhile, it is important to note that the correlation was not perfect: sequences of $P$. aleyrodidarum from MEAM1 and MED were assigned to the same clade instead of the two distinct clades presented in the mtCO1 tree. The reason could be the dissemination of the MEAM1 and MED species; furthermore, the spread of these two invasive species in China has been frequently associated with founder effects that fix specific mtDNA variation(s) along with particular endosymbionts (Chu et al. 2011; Gueguen et al. 2010). Taken together, although there was similarity between the two trees of P. aleyrodidarum and $B$. tabaci, the genetic variation of primary symbiont might not be an ideal reflecting the genetic variation of the cryptic B. tabaci.

The S-endosymbionts, Arsenophonus and Cardinium, both showed a lack of congruence with 
234 the B. tabaci mtCO1 phylogram. This is consistent with the finding from Ahmed et al. (2013), who 235 provided evidence for horizontal transmission of S-endosymbionts in the B. tabaci cryptic species 236 complex based on phylogenies studies. There are substantial phylogenetic evidences showing that

237 S-endosymbionts such as Wolbachia and Arsenophonus, undergoing horizontal transfer among 238 host arthropod species (Ahmed et al. 2013; Chrostek et al. 2017; Kolasa et al. 2017; Li et al. 2017; 239 Russell et al. 2003; Vavre et al. 1999). In some cases, the mechanisms for horizontal transmission 240 of S-endosymbionts are already known, including transferring through parasitoid wasps (Ahmed 241 et al. 2015; Gehrer \& Vorburger 2012), plants (Caspi-Fluger et al. 2012; Li et al. 2017) or even 242 sexual transmission (Moran \& Dunbar 2006). Therefore, the potential horizontal transfer of S243 endosymbionts in our samples could be one or combination of the above ways.

In summary, this study reported the varied prevalence of three endosymbionts within five $B$. tabaci cryptic species. The P-endosymbiont $P$. aleyrodidarum was detected in all whitefly individuals; the S-endosymbionts Arsenophonus was abundant in native species while Cardinium was common in the invasive species. In addition, the phylogenetic relationships between endosymbionts and their hosts $B$. tabaci probably indicated the vertical transmission and coevolution of $P$. aleyrodidarum and B. tabaci; meanwhile, horizontal transfer of Arsenophonus and Cardinium may happen in our collecting samples. Our study not only reported current infection status of endosymbionts within B. tabaci populations in China, but also demonstrated that Sendosymbionts genetic variation may not reflect host genetic variation and should not be used to infer taxonomic relationships within the host species complex. If funding allows, more endosymbionts should be investigated and future investigations could be the contribution of endosymbionts to invasiveness, population expansion, or even competitiveness of whitefly species.

\section{Acknowledgements}

We express our deep gratitude to the Testing Center of Yangzhou University. We sincerely thank Dr. Kerry M. Oliver from Department of Entomology, University of Georgia for polishing 
261

262

263

264

265

266

267

268

269

270

271

272

273

274

275

276

277

278

279

280

281

282

283

284

285

286

287

288

289

290

291

292

293

294

295

296

297

298

299

the manuscript and providing valuable comments.

\section{References}

Ahmed MZ, De Barro PJ, Ren SX, Greeff JM, and Qiu BL. 2013. Evidence for horizontal transmission of secondary endosymbionts in the Bemisia tabaci cryptic species complex. PloS one 8:e53084.

Ahmed MZ, Li SJ, Xue X, Yin XJ, Ren SX, Jiggins FM, Greeff JM, and Qiu BL. 2015. The intracellular bacterium Wolbachia uses parasitoid wasps as phoretic vectors for efficient horizontal transmission. PLoS Pathog 11:e1004672.

Ahmed MZ, Ren SX, Mandour NS, Greeff JM, and Qiu BL. 2010. Prevalence of Wolbachia supergroups A and B in Bemisia tabaci (Hemiptera: Aleyrodidae) and some of its natural enemies. Journal of economic entomology 103:1848-1859.

Baumann P. 2005. Biology of bacteriocyte-associated endosymbionts of plant sap-sucking insects. Annu Rev Microbiol 59:155-189.

Bing XL, Ruan YM, Rao Q, Wang XW, and Liu SS. 2013. Diversity of secondary endosymbionts among different putative species of the whitefly Bemisia tabaci. Insect Science 20:194206.

Bing XL, Xia WQ, Gui JD, Yan GH, Wang XW, and Liu SS. 2014. Diversity and evolution of the Wolbachia endosymbionts of Bemisia (Hemiptera: Aleyrodidae) whiteflies. Ecology and evolution 4:2714-2737.

Brown J. 1994. Current status of Bemisia tabaci as a plant pest and virus vector in agroecosystems worldwide. FAO Plant Protection Bulletin 42:3-32.

Caspi-Fluger A, Inbar M, Mozes-Daube N, Katzir N, Portnoy V, Belausov E, Hunter MS, and Zchori-Fein E. 2012. Horizontal transmission of the insect symbiont Rickettsia is plantmediated. Proc R Soc B 279:1791-1796.

Caspi-Fluger A, Inbar M, Mozes-Daube N, Mouton L, Hunter MS, and Zchori-Fein E. 2011. Rickettsia 'in' and 'out': two different localization patterns of a bacterial symbiont in the same insect species. PloS one 6:e21096.

Chenna R, Sugawara H, Koike T, Lopez R, Gibson TJ, Higgins DG, and Thompson JD. 2003. Multiple sequence alignment with the Clustal series of programs. Nucleic acids research 31:3497-3500.

Chiel E, Gottlieb Y, Zchori-Fein E, Mozes-Daube N, Katzir N, Inbar M, and Ghanim M. 2007. Biotype-dependent secondary symbiont communities in sympatric populations of Bemisia tabaci. Bulletin of entomological research 97:407-413.

Chrostek E, Pelz-Stelinski K, Hurst GD, and Hughes GL. 2017. Horizontal transmission of intracellular insect symbionts via plants. Frontiers in Microbiology 8.

Chu D, Gao C, De Barro P, Zhang Y, Wan F, and Khan I. 2011. Further insights into the strange role of bacterial endosymbionts in whitefly, Bemisia tabaci: Comparison of secondary 
300

301

302

303

304

305

306

307

308

309

310

311

312

313

314

315

316

317

318

319

320

321

322

323

324

325

326

327

328

329

330

331

332

333

334

335

336

337

338

339

340

symbionts from biotypes B and Q in China. Bulletin of entomological research 101:477486.

Chu D, Zhang Y, Cong B, Xu B, and Wu Q. 2005. Identification for Yunnan Q-biotype Bemisia tabaci population. Entomological Knowledge 42:59-62.

Cicero J, Fisher T, and Brown JK. 2016. Localization of 'Candidatus Liberibacter solanacearum'and Evidence for Surface Appendages in the Potato Psyllid Vector. Phytopathology 106:142-154.

Cohen S, and Nitzany F. 1966. Transmission and host range of the tomato yellow leaf curl virus. Phytopathology 56.

Cooper WR, Sengoda VG, and Munyaneza JE. 2014. Localization of 'Candidatus Liberibacter solanacearum' (Rhizobiales: Rhizobiaceae) in Bactericera cockerelli (Hemiptera: Triozidae). Annals of the Entomological Society of America 107:204-210.

De Barro PJ, Liu SS, Boykin LM, and Dinsdale AB. 2011. Bemisia tabaci: a statement of species status. Annual review of entomology 56:1-19.

Dinsdale A, Cook L, Riginos C, Buckley Y, and De Barro P. 2010. Refined global analysis of Bemisia tabaci (Hemiptera: Sternorrhyncha: Aleyrodoidea: Aleyrodidae) mitochondrial cytochrome oxidase 1 to identify species level genetic boundaries. Annals of the Entomological Society of America 103:196-208.

Dobson SL, Bourtzis K, Braig HR, Jones BF, Zhou W, Rousset F, and O'Neill SL. 1999. Wolbachia infections are distributed throughout insect somatic and germ line tissues. Insect biochemistry and molecular biology 29:153-160.

Douglas A. 1998. Nutritional interactions in insect-microbial symbioses: aphids and their symbiotic bacteria Buchnera. Annual review of entomology 43:17-37.

Everett KD, Thao M, Horn M, Dyszynski GE, and Baumann P. 2005. Novel chlamydiae in whiteflies and scale insects: endosymbionts 'Candidatus Fritschea bemisiae'strain Falk and 'Candidatus Fritschea eriococci'strain Elm. International journal of systematic and evolutionary microbiology 55:1581-1587.

Gehrer L, and Vorburger C. 2012. Parasitoids as vectors of facultative bacterial endosymbionts in aphids. Biology letters:rsbl20120144.

Ghosh S, Bouvaine S, and Maruthi M. 2015. Prevalence and genetic diversity of endosymbiotic bacteria infecting cassava whiteflies in Africa. BMC microbiology 15:93.

Ghosh S, Bouvaine S, Richardson SC, Ghanim M, and Maruthi M. 2018. Fitness costs associated with infections of secondary endosymbionts in the cassava whitefly species Bemisia tabaci. Journal of pest science 91:17-28.

Gosalbes MJ, Latorre A, Lamelas A, and Moya A. 2010. Genomics of intracellular symbionts in insects. International Journal of Medical Microbiology 300:271-278.

Gottlieb Y, Ghanim M, Gueguen G, Kontsedalov S, Vavre F, Fleury F, and Zchori-Fein E. 2008. Inherited intracellular ecosystem: symbiotic bacteria share bacteriocytes in whiteflies. The FASEB Journal 22:2591-2599.

Gueguen G, Vavre F, Gnankine O, Peterschmitt M, Charif D, Chiel E, Gottlieb Y, Ghanim M, ZCHORI - FEIN E, and Fleury F. 2010. Endosymbiont metacommunities, mtDNA 
diversity and the evolution of the Bemisia tabaci (Hemiptera: Aleyrodidae) species complex. Molecular Ecology 19:4365-4376.

Hashmi TR, Devi SR, Meshram NM, and Prasad R. 2018. Assessment of bacterial endosymbionts and the host, Bemisia tabaci (Hemiptera: Aleyrodidae), using rRNA and mitochondrial cytochrome oxidase I gene sequences. Communicative \& Integrative Biology 11:e1433442.

Hu J, Zhang X, Jiang Z, Zhang F, Liu Y, Li Z, and Zhang Z. 2018. New putative cryptic species detection and genetic network analysis of Bemisia tabaci (Hempitera: Aleyrodidae) in China based on mitochondrial COI sequences. Mitochondrial DNA Part A 29:474-484.

Jahan S, Lee K, Howlader M, Bashar H, and Hasan G. 2015. Molecular divergence of secondary endosymbiont, Cardinium in Bemisia tabaci (Gennadius) and associates. Bangladesh Journal of Agricultural Research 40:121-135.

Karut K, Mete Karaca M, Döker İ, and Kazak C. 2017. Analysis of Species, Subgroups, and Endosymbionts of Bemisia tabaci (Hemiptera: Aleyrodidae) From Southwestern Cotton Fields in Turkey. Environmental entomology.

Karut K, and Tok B. 2014. Secondary endosymbionts of Turkish Bemisia tabaci (Gennadius) populations. Phytoparasitica 42:413-419.

Kolasa M, Montagna M, Mereghetti V, Kubisz D, Mazur MA, and Kajtoch Ł. 2017. Preliminary evidence of the horizontal transmission of Wolbachia between Crioceris leaf beetles (Coleoptera: Chrysomelidae) and their Asparagus host plants. European Journal of Entomology 114:446-454.

Li SJ, Ahmed MZ, Lv N, Shi PQ, Wang XM, Huang JL, and Qiu BL. 2017. Plantmediated horizontal transmission of Wolbachia between whiteflies. The ISME journal 11:1019.

Liu S-S, De Barro P, Xu J, Luan J-B, Zang LS, Ruan YM, and Wan FH. 2007. Asymmetric mating interactions drive widespread invasion and displacement in a whitefly. Science 318:17691772.

Luo C, Yao Y, Wang R, Yan F, Hu D, and Zhang Z. 2002. The use of mitochondrial cytochrome oxidase I (mt CO I) gene sequences for the identification of biotypes of Bemisia tabaci (Gennadius) in China. Kun chong xue bao Acta entomologica Sinica 45:757-763.

Marubayashi JM, Kliot A, Yuki VA, Rezende JAM, Krause-Sakate R, Pavan MA, and Ghanim M. 2014. Diversity and localization of bacterial endosymbionts from whitefly species collected in Brazil. PloS one 9:e108363.

Morag N, Klement E, Saroya Y, Lensky I, and Gottlieb Y. 2012. Prevalence of the symbiont Cardinium in Culicoides (Diptera: Ceratopogonidae) vector species is associated with land surface temperature. The FASEB Journal 26:4025-4034.

Moran NA, and Baumann P. 2000. Bacterial endosymbionts in animals. Current opinion in microbiology 3:270-275.

Moran NA, and Dunbar HE. 2006. Sexual acquisition of beneficial symbionts in aphids. Proceedings of the National Academy of Sciences 103:12803-12806.

Pan H, Li X, Ge D, Wang S, Wu Q, Xie W, Jiao X, Chu D, Liu B, and Xu B. 2012. Factors affecting population dynamics of maternally transmitted endosymbionts in Bemisia tabaci. 
PloS one 7:e30760.

Parrella G, Nappo AG, Manco E, Greco B, and Giorgini M. 2014. Invasion of the Q2 mitochondrial variant of Mediterranean Bemisia tabaci in southern Italy: possible role of bacterial endosymbionts. Pest management science 70:1514-1523.

Posada D, and Crandall KA. 1998. Modeltest: testing the model of DNA substitution. Bioinformatics (Oxford, England) 14:817-818.

Qiu B, Coats SA, Shunxiang R, Idris AM, Caixia X, and Brown JK. 2007. Phylogenetic relationship of native and introduced Bemisia tabaci (Homoptera: Aleyrodidae) from China and India based on mtCOI DNA sequencing and host plant comparisons. Progress in Natural Science 17:645-654.

Rao Q, Rollat-Farnier PA, Zhu DT, Santos-Garcia D, Silva FJ, Moya A, Latorre A, Klein CC, Vavre F, and Sagot MF. 2015. Genome reduction and potential metabolic complementation of the dual endosymbionts in the whitefly Bemisia tabaci. BMC genomics 16:226.

Ronquist F, and Huelsenbeck JP. 2003. MrBayes 3: Bayesian phylogenetic inference under mixed models. Bioinformatics 19:1572-1574.

Russell J, Latorre A, Sabater - Muñoz B, Moya A, and Moran N. 2003. Side - stepping secondary symbionts: widespread horizontal transfer across and beyond the Aphidoidea. Molecular Ecology 12:1061-1075.

Santos-Garcia D, Vargas-Chavez C, Moya A, Latorre A, and Silva FJ. 2015. Genome evolution in the primary endosymbiont of whiteflies sheds light on their divergence. Genome biology and evolution 7:873-888.

Simon C, Frati F, Beckenbach A, Crespi B, Liu H, and Flook P. 1994. Evolution, weighting, and phylogenetic utility of mitochondrial gene sequences and a compilation of conserved polymerase chain reaction primers. Annals of the Entomological Society of America 87:651-701.

Skaljac M, Zanic K, Ban SG, Kontsedalov S, and Ghanim M. 2010. Co-infection and localization of secondary symbionts in two whitefly species. BMC microbiology 10:142.

Sseruwagi P, Wainaina J, Ndunguru J, Tumuhimbise R, Tairo F, Guo J-Y, Vrielink A, Blythe A, Kinene T, and De Marchi B. 2018. The first transcriptomes from field-collected individual whiteflies (Bemisia tabaci, Hemiptera: Aleyrodidae): a case study of the endosymbiont composition. Gates open research 1.

Sun DB, Liu YQ, Qin L, Xu J, Li FF, and Liu SS. 2013. Competitive displacement between two invasive whiteflies: insecticide application and host plant effects. Bulletin of entomological research 103:344-353.

Thao ML, and Baumann P. 2004. Evolutionary relationships of primary prokaryotic endosymbionts of whiteflies and their hosts. Applied and environmental microbiology 70:3401-3406.

Thierry M, Becker N, Hajri A, Reynaud B, LETT JM, and Delatte H. 2011. Symbiont diversity and non - random hybridization among indigenous (Ms) and invasive (B) biotypes of 
421 Bemisia tabaci. Molecular Ecology 20:2172-2187.

422 Thierry M, Bile A, Grondin M, Reynaud B, Becker N, and Delatte H. 2015. Mitochondrial, 423 nuclear, and endosymbiotic diversity of two recently introduced populations of the invasive

424

425

426

427

428

429

430

431

432

433

434

435

436

437

438

439

440

441

442

443

444

445

446

447

448

449

450

451

452

453
Bemisia tabaci MED species in La Réunion. Insect Conservation and Diversity 8:71-80.

Vavre F, Fleury F, Lepetit D, Fouillet P, and Boulétreau M. 1999. Phylogenetic evidence for horizontal transmission of Wolbachia in host-parasitoid associations. Molecular biology and evolution 16:1711-1723.

Wang XW, Li P, and Liu SS. 2017. Whitefly interactions with plants. Curr Opin Insect Sci 19:7075. 10.1016/j.cois.2017.02.001

Werren JH, and O'Neill SL. 1997. The evolution of heritable symbionts. Influential passengers: inherited microorganisms and arthropod reproduction:1-41.

Zchori-Fein E, Lahav T, and Freilich S. 2014. Variations in the identity and complexity of endosymbiont combinations in whitefly hosts. Frontiers in Microbiology 5:310.

Zchori-Fein E, Roush RT, and Rosen D. 1998. Distribution of parthenogenesis-inducing symbionts in ovaries and eggs of Aphytis (Hymenoptera: Aphelinidae). Current microbiology 36:1-8.

\section{7}

(1)

(1)

0

1

2

3

4

45

46

47

48

49

.




\section{Figure Captions}

457

458

459

460

461

462

463

464

465

466

467

468

469

470

471

472

473

474

Figure 1. The quantity and distribution of $B$. tabaci cryptic species in China. (A) The quantity

of each B. tabaci cryptic species based on molecular identification. (B) The locations of the $B$. tabaci cryptic species populations in China. Names of locations are given in Table S1. Maps were created using Esri’s ArcGIS platform (http://www.esri.com/software/arcgis).

Figure 2. Infection frequency of endosymbionts in five $B$. tabaci cryptic species. (A) $P$. aleyrodidarum; (B) Arsenophonus; (C) Cardinium. Number above bars indicate the number of infection.

Figure 3. The Bayesian phylogenetic tree of $B$. tabaci cryptic species based on mtCOI sequences. The value beside the nodes are posterior probabilities. Trialeurodes vaporariorum (AF418672) is used as outgroup. Accession numbers for $m t C O I$ sequences submitted to GenBank are KP137471-KP137491. All $m t C O I$ sequences of $B$. tabaci cryptic species used in this study were clustered with other related references sequences from GenBank and their accession numbers are also indicated in the tree. Bold dots indicate the sequences from the present study. 
475 used as outgroup. Accession numbers for $16 \mathrm{~S}$ rDNA sequences submitted to GenBank are 476 KP201110-KP201125. All 16S rDNA sequences of $P$. aleyrodidarum used in this study were 477 clustered with other related references sequences from GenBank and their accession numbers are 478 also indicated in the tree. Bold dots indicate the sequences from the present study. Dotted boxes 479 indicate imperfect cluster of each B. tabaci cryptic species.

Figure 5. The Bayesian phylogenetic tree of Arsenophonus based on 23S $r D N A$ sequences.

The value beside the nodes are posterior probabilities. Aleurodicus dispersus (AY264664) is used as outgroup. Accession numbers for 23S rDNA sequences submitted to GenBank are KP201103KP201109. All 23S rDNA sequences of Arsenophonus used in this study were clustered with other related references sequences from GenBank and their accession numbers are also indicated in the tree. A1-A4 indicate the four clusters.

Figure 6. The Bayesian phylogenetic tree of Cardinium based on $16 \mathrm{~S} r D N A$ sequences. The value beside the nodes are posterior probabilities. Paralvinella palmiformis (AJ441237) is used as outgroup. Accession numbers for 16S rDNA sequences submitted to GenBank are KP201127KP201134. All 16S rDNA sequences of Cardinium used in this study were clustered with other related references sequences from GenBank and their accession numbers are also indicated in the tree. C1-C3 indicate the three clusters. 
Figure 1

The quantity and distribution of $B$. tabaci cryptic species in China.

(A) The quantity of each $B$. tabaci cryptic species based on molecular identification. (B) The locations of the $B$. tabaci cryptic species populations in China. Names of locations are given in Table S1. Maps were created using Esri's ArcGIS platform ( http://www.esri.com/software/arcgis) .

A

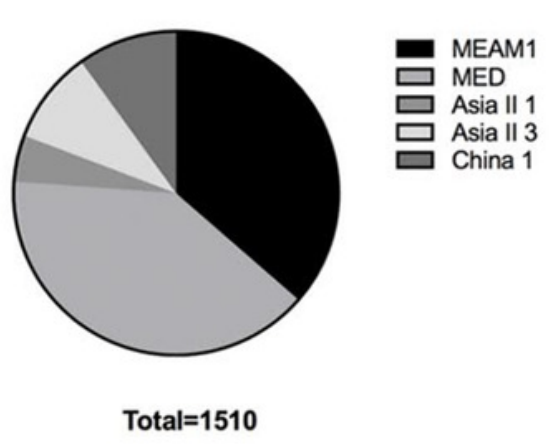

B

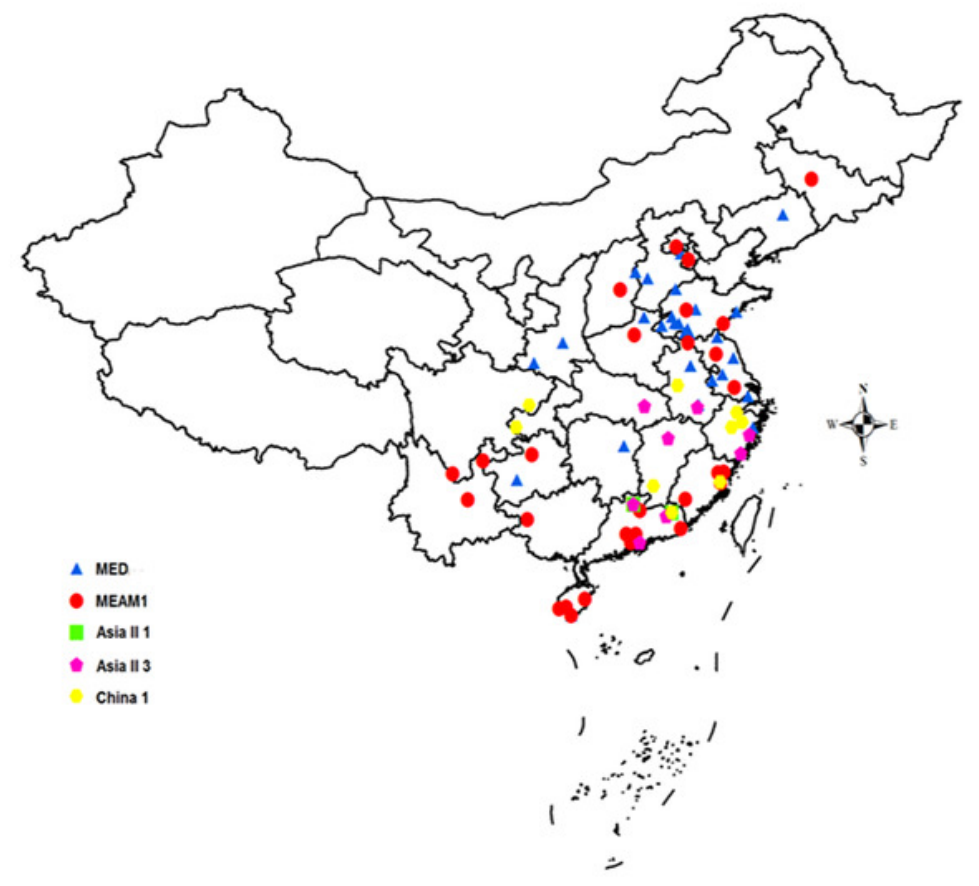


Figure 2

Infection frequency of endosymbionts in five $B$. tabaci cryptic species.

(A) P. aleyrodidarum; (B) Arsenophonus; (C) Cardinium. Number above bars indicate the number of infection.
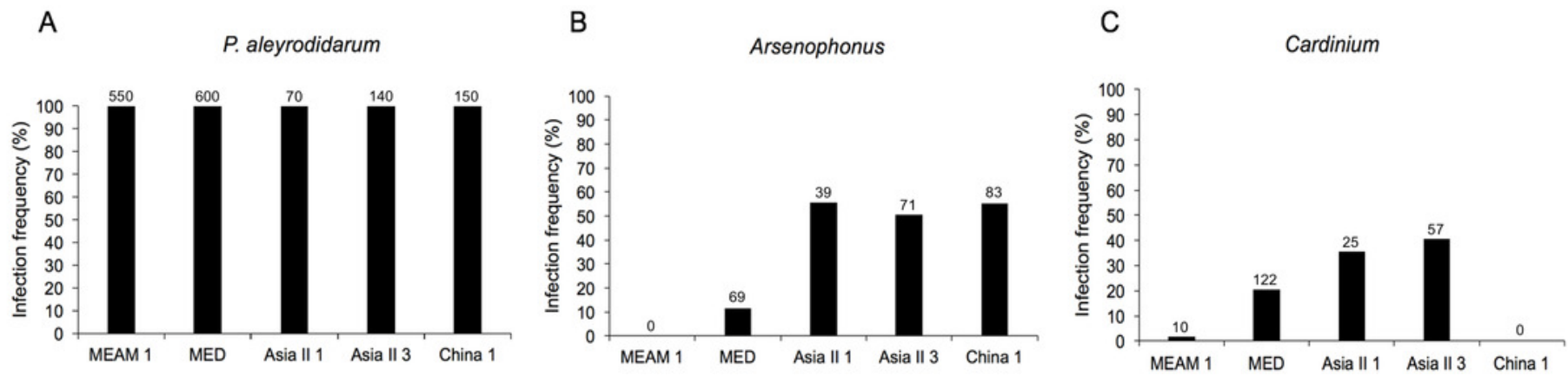


\section{Figure 3}

The Bayesian phylogenetic tree of $B$. tabaci cryptic species based on $m t C O l$ sequences.

The value beside the nodes are posterior probabilities. Trialeurodes vaporariorum

(AF418672) is used as outgroup. Accession numbers for $m t C O$ sequences submitted to

GenBank are KP137471-KP137491. All mtCOI sequences of B. tabaci cryptic species used in this study were clustered with other related references sequences from GenBank and their accession numbers are also indicated in the tree. Bold dots indicate the sequences from the present study. 


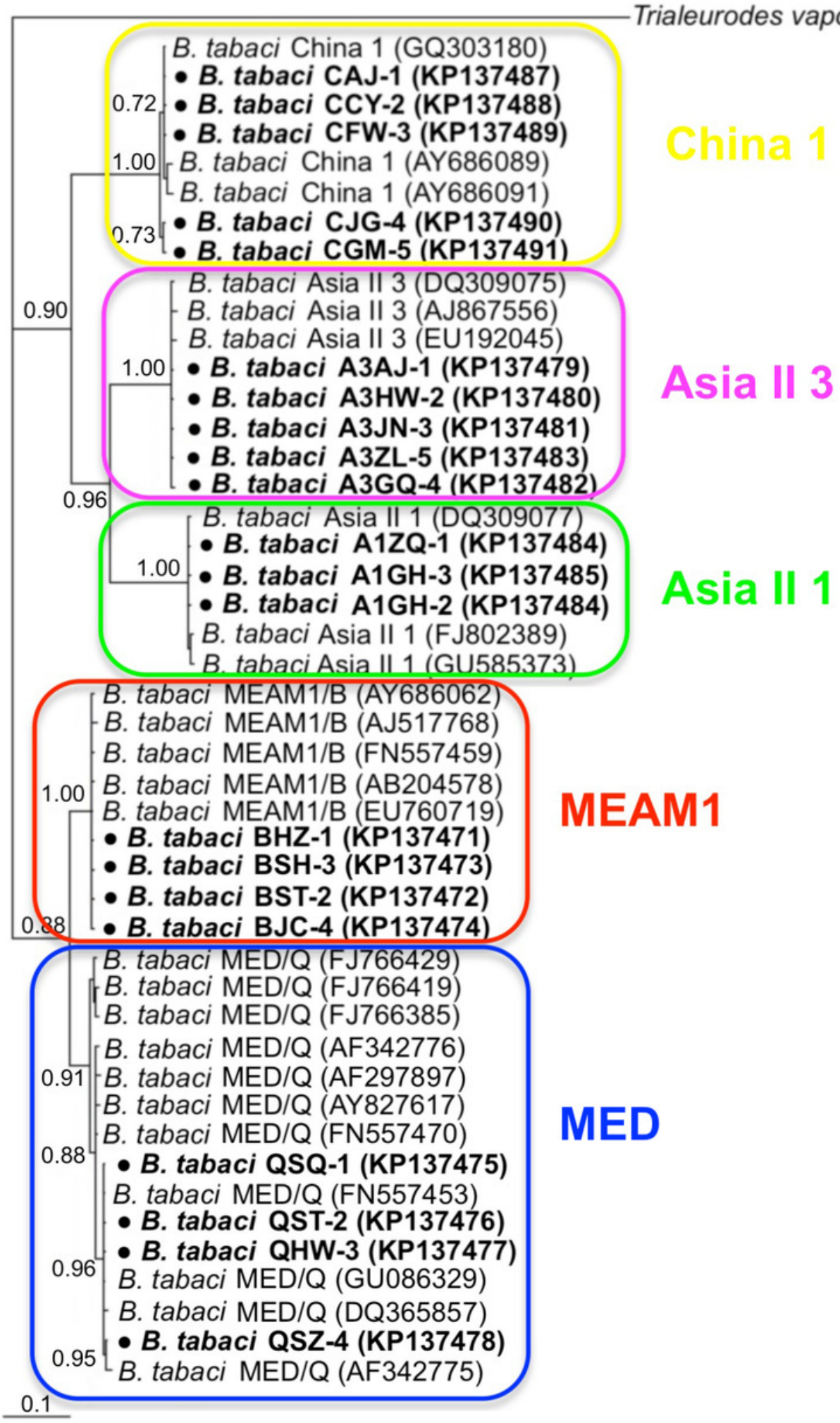




\section{Figure 4}

The Bayesian phylogenetic tree of $P$. aleyrodidarum based on $16 \mathrm{~S} r \mathrm{DNA}$ sequences.

The value beside the nodes are posterior probabilities. Trialeurodes vaporariorum

(AY266113) is used as outgroup. Accession numbers for $16 \mathrm{~S}$ rDNA sequences submitted to GenBank are KP201110-KP201125. All 16S rDNA sequences of $P$. aleyrodidarum used in this study were clustered with other related references sequences from GenBank and their accession numbers are also indicated in the tree. Bold dots indicate the sequences from the present study. Dotted boxes indicate imperfect cluster of each B. tabaci cryptic species. 
- B. tabaci MED/Q (KP201113)

- B. tabaci China 1 (KP201124)

- B. tabaci China 1 (KP201125)

- B. tabaci China 1 (KP201126)

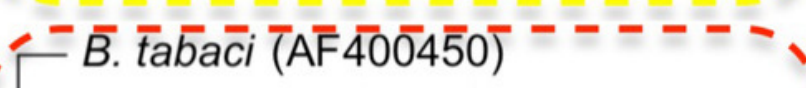

- B. tabaci MGAM1/B (KP201111)

- B. tabaci MED/Q (KP201114)

[ B. tabaci (AF400463)

B. tabaci (AF400465)

1.o0 Buchnera aphidicola (M63246)

- Bemisia argentifolii (AF211870)

B. tabaci MGAM1/B (KP201110)

- B. tabaci MED/Q (KP201116)

- B. tabaci MED/Q (KP201117)

B. tabaci MED/Q (KP201112)

- B. tabaci Asia II 3 (KP201119)

- B. tabaci MED/Q (KP201115)

Q.92 • B. tabaci China 1 (KP201123)

B. tabaci Asia I (JN204487)

B. tabaci Asia II 1 (JN204493)

- B. tabaci Asia II 1 (KP201122)

094

B. tabaci (AF400457)

B. tabaci Asia I (JN204489)

-B. tabaci Asia II 1(JN204486)

- B. tabaci Asia II 1 (KP201121)

- B. tabaci Asia II 3 (KP201118)

0.89

B. tabaci Asia II 3 (KP201120)
MEAM1 + MED

\section{Asia II 1}

\section{Asia II 3}




\section{Figure 5}

The Bayesian phylogenetic tree of Arsenophonus based on 235 rDNA sequences.

The value beside the nodes are posterior probabilities. Aleurodicus dispersus (AY264664) is used as outgroup. Accession numbers for $23 \mathrm{~S}$ rDNA sequences submitted to GenBank are KP201103-KP201109. All 23S rDNA sequences of Arsenophonus used in this study were clustered with other related references sequences from GenBank and their accession numbers are also indicated in the tree. A1-A4 indicate the four clusters. 


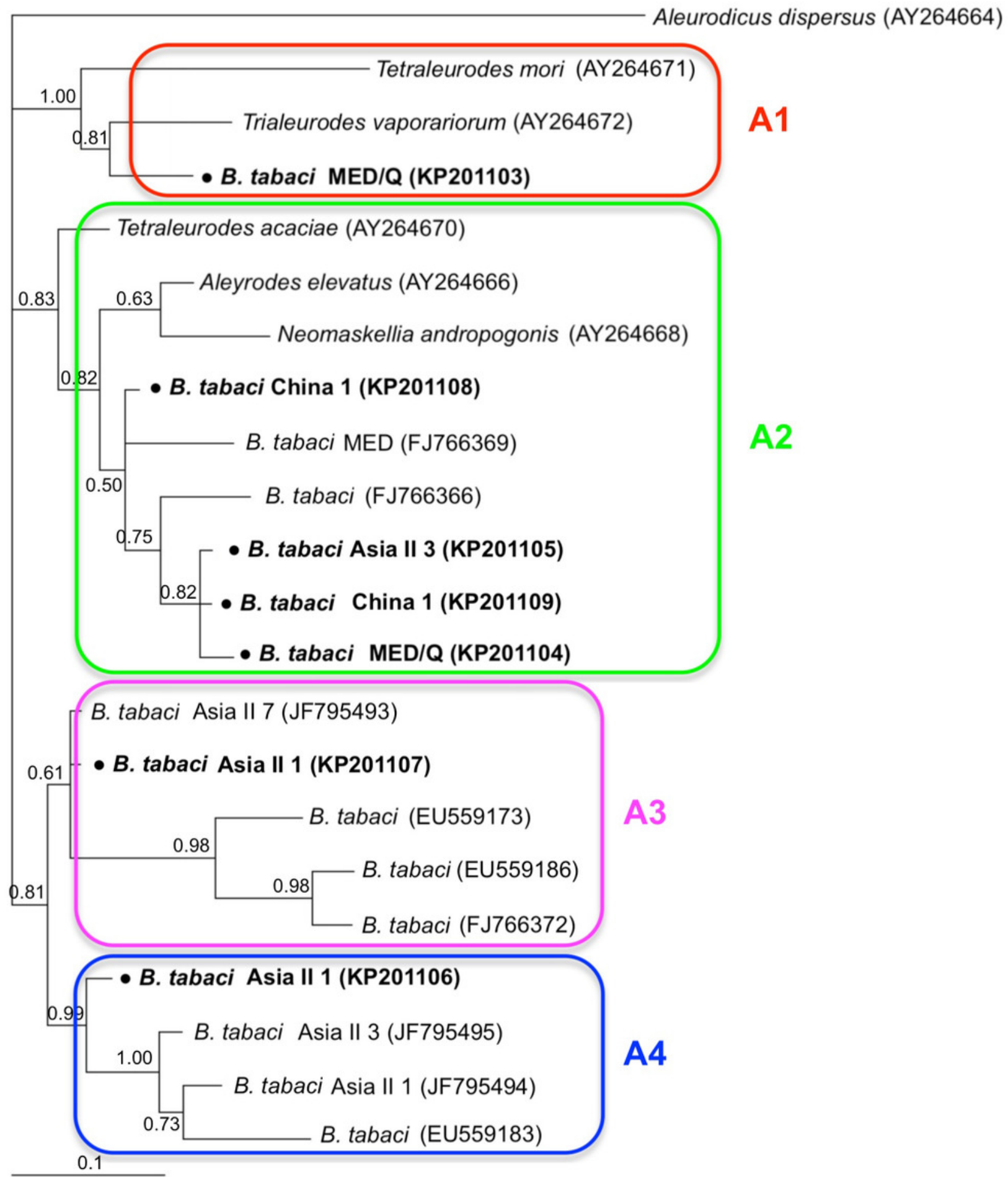




\section{Figure 6}

The Bayesian phylogenetic tree of Cardinium based on 16S rDNA sequences.

The value beside the nodes are posterior probabilities. Paralvinella palmiformis (AJ441237) is used as outgroup. Accession numbers for $16 \mathrm{~S}$ rDNA sequences submitted to GenBank are KP201127-KP201134. All 16S rDNA sequences of Cardinium used in this study were clustered with other related references sequences from GenBank and their accession numbers are also indicated in the tree. $\mathrm{C} 1-\mathrm{C} 3$ indicate the three clusters. 


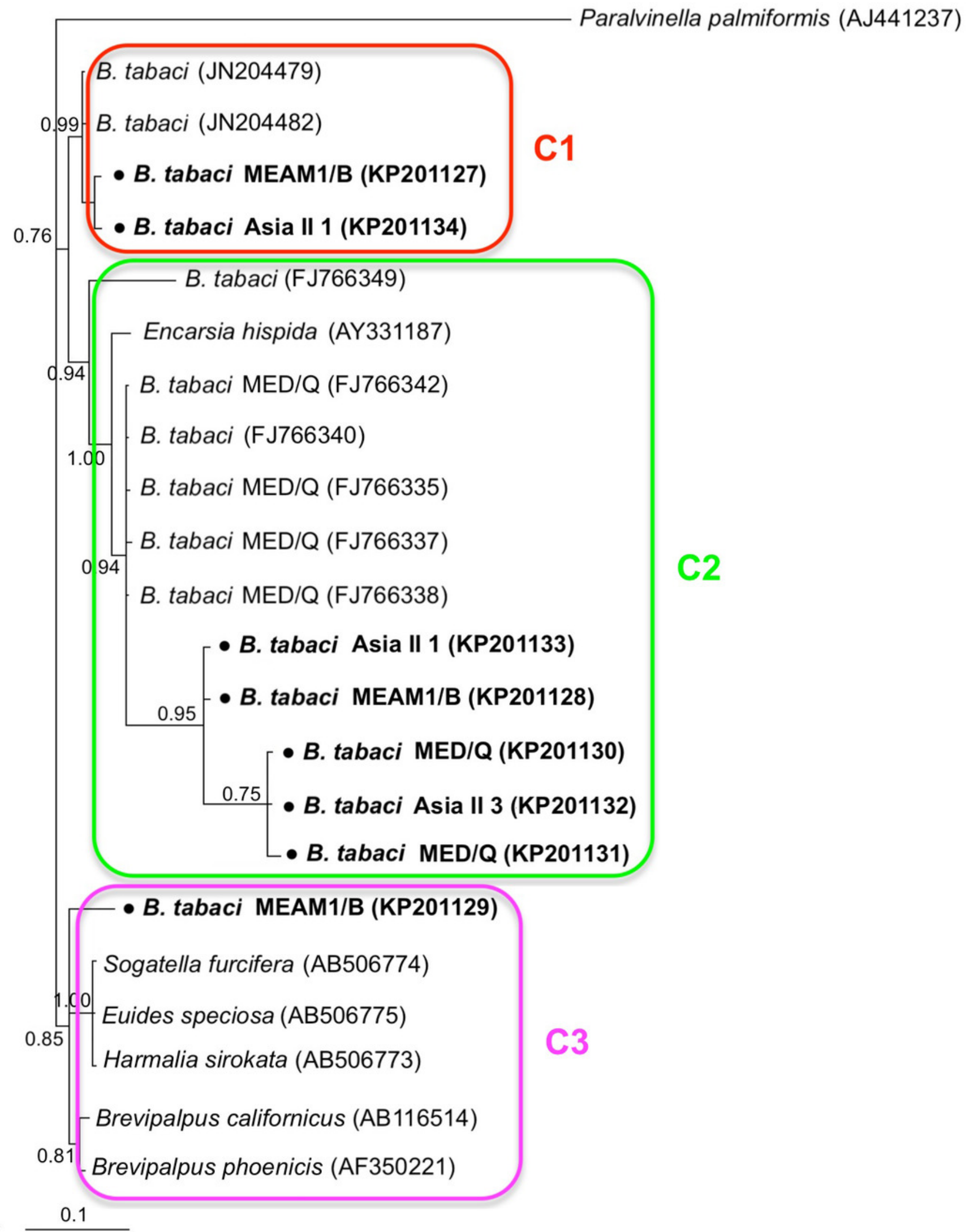




\section{Table $\mathbf{1}$ (on next page)}

The sequences, annealing temperature, product size and references of primers used in this study. 
1 Table 1. The sequences, annealing temperature, product size and references of primers used in this study.

2

\begin{tabular}{|c|c|c|c|c|}
\hline Gene & Primer sequence $\left(5^{\prime} \rightarrow 3^{\prime}\right)$ & $\begin{array}{c}\text { Annealing } \\
\text { temperature }\end{array}$ & $\begin{array}{l}\text { Product } \\
\text { size(bp) }\end{array}$ & References \\
\hline B. tabaci mtCOI & $\begin{array}{l}\text { C1-J-2195: TTGATTTTTTGGTCATCCAGAAGT } \\
\text { L2-N-3014: TCCAATGCACTAATCTGCCATATTA }\end{array}$ & $50^{\circ} \mathrm{C}$ & 800 & Frohlich et al., 1999 \\
\hline $\begin{array}{l}\text { P. aleyrodidarum } \\
\quad 16 \mathrm{~S} \text { rDNA }\end{array}$ & $\begin{array}{l}\text { Pro-F: TGCAAGTCGAGCGGCATCAT } \\
\text { Pro-R: AAAGTTCCCGCCTTATGCGT }\end{array}$ & $59^{\circ} \mathrm{C}$ & 1000 & Zghori-Fein and Brown, 2002 \\
\hline $\begin{array}{l}\text { Cardinium } 16 S \\
\quad \text { rDNA }\end{array}$ & $\begin{array}{l}\text { Ch-F: TACTGTAAGAAATAAGCACCGGC } \\
\text { Ch-R: GTGGATCACTTAACGCTTTCG }\end{array}$ & $57^{\circ} \mathrm{C}$ & 400 & Zchori-Fein et al., 2004 \\
\hline $\begin{array}{l}\text { Arsenophonus } 23 S \\
\text { rDNA } \\
\end{array}$ & $\begin{array}{l}\text { Ars-F: CGTTTGATGAATTCATAGTCAAA } \\
\text { Ars-R: GGTCCTCCAGTTAGTGTTACCCAAC }\end{array}$ & $60.5^{\circ} \mathrm{C}$ & 900 & Thao and Baumann, 2004 \\
\hline
\end{tabular}




\section{Table 2 (on next page)}

Haplotype information of whitefly, P. aleyrodidarum, Arsenophonus and Cardinium. 
1 Table 2 Haplotype information of whitefly, P. aleyrodidarum, Arsenophonus and Cardinium.

\begin{tabular}{|c|c|c|c|c|c|c|c|c|c|c|c|c|c|c|c|c|}
\hline \multirow[b]{2}{*}{ Species } & \multirow[b]{2}{*}{$\mathrm{n}$. } & \multicolumn{2}{|c|}{$\mathrm{mt} \mathrm{COI}$} & \multirow[b]{2}{*}{ Per.(\%) } & \multicolumn{4}{|c|}{ P.aleyrodidarum } & \multicolumn{4}{|c|}{ Arsenophonus } & \multicolumn{4}{|c|}{ Cardinium } \\
\hline & & Re.Seq. & Acc. no. & & n. & Re.Seq. & Acc. no. & Per.(\%) & $\mathrm{n}$. & Re.Seq. & Acc. no. & Per.(\%) & n. & Re.Seq. & Acc. no. & Per.(\%) \\
\hline \multirow[t]{4}{*}{ MEAM1/B } & 4 & BHZ-1 & KP137471 & 85.1 & 2 & BST-CA1 & KP201110 & 89.1 & & & & & 3 & BGD-CR1 & KP201127 & 92.5 \\
\hline & & BST-2 & KP137472 & 8.2 & & BGW-CA2 & KP201111 & 10.9 & & & & & & BHC-CR2 & KP201128 & 2.5 \\
\hline & & BSH-3 & KP137473 & 4.9 & & & & & & & & & & BSRF-CR3 & KP201129 & 5 \\
\hline & & BJC-4 & KP137474 & 1.8 & & & & & & & & & & & & \\
\hline \multirow[t]{6}{*}{ MED/Q } & 4 & QSQ-1 & KP137475 & 90.3 & 6 & QBJ-CA1 & KP201112 & 88.8 & 2 & QHS-AR1 & KP201103 & 89.7 & 2 & QSH-CR1 & KP201130 & 96.2 \\
\hline & & QST-2 & KP137476 & 0.5 & & QAH-CA2 & KP201113 & 5.8 & & QHL-AR2 & KP201104 & 10.3 & & QSZ-CR2 & KP201131 & 0.4 \\
\hline & & QHW-3 & KP137477 & 5.8 & & QSL-CA3 & KP201114 & 1.0 & & & & & & & & \\
\hline & & QSZ-4 & KP137478 & 3.3 & & QSJ-CA4 & KP201115 & 2.0 & & & & & & & & \\
\hline & & & & & & QSH-CA5 & KP201116 & 1.8 & & & & & & & & \\
\hline & & & & & & QAH-CA6 & KP201117 & 0.5 & & & & & & & & \\
\hline \multirow[t]{5}{*}{ Aisa II 3} & 5 & A3AJ-1 & KP137479 & 86.9 & 3 & A3ZW-CA1 & KP201118 & 86.2 & 1 & A3ZL-AR1 & KP201105 & 100.0 & 1 & A3ZL-CR1 & KP201132 & 100.0 \\
\hline & & A3HW-2 & KP137480 & 1.5 & & A3ZL-CA2 & KP201119 & 9.2 & & & & & & & & \\
\hline & & A3JN-3 & KP137481 & 0.8 & & A3GQ-CA3 & KP201120 & 4.6 & & & & & & & & \\
\hline & & A3GQ-4 & KP137482 & 6.2 & & & & & & & & & & & & \\
\hline & & A3ZL-5 & KP137483 & 4.6 & & & & & & & & & & & & \\
\hline \multirow[t]{3}{*}{ Aisa II 1} & 3 & A1ZQ-1 & KP137484 & 81.4 & 2 & A1GQ-CA1 & KP201121 & 94.3 & 2 & A1ZL-AR1 & KP201106 & 92.3 & 2 & A1GQ-CR1 & KP201133 & 92.0 \\
\hline & & A1GH-2 & KP137485 & 12.9 & & A1JS-CA2 & KP201122 & 5.7 & & A1GS-AR2 & KP201107 & 7.7 & & A1SL-CR2 & KP201134 & 8.0 \\
\hline & & A1GH-3 & KP137486 & 5.7 & & & & & & & & & & & & \\
\hline \multirow[t]{5}{*}{ China 1} & 5 & CAJ-1 & KP137487 & 94.7 & 4 & CCY-CA1 & KP201123 & 2.7 & 2 & CCY-AR1 & KP201108 & 97.6 & & & & \\
\hline & & $\mathrm{CCY}-2$ & KP137488 & 2.0 & & CZS-CA2 & KP201124 & 90.7 & & CAJ-AR2 & KP201109 & 2.4 & & & & \\
\hline & & CFW-3 & KP137489 & 0.7 & & CZH-CA3 & KP201125 & 5.3 & & & & & & & & \\
\hline & & CJG-4 & KP137490 & 1.3 & & CZJ-CA4 & KP201126 & 1.3 & & & & & & & & \\
\hline & & CGM-5 & KP137491 & 1.3 & & & & & & & & & & & & \\
\hline
\end{tabular}

